\title{
Modelo con autómatas celulares para analizar la accesibilidad peatonal al interior del campus universitario Meléndez de la Universidad del Valle
}

\author{
Cellular automata model to analyze the pedestrian accessibility within the \\ Meléndez campus of the University of Valle
}

\author{
V. M. Vargas-Forero (iD , L. E. Muñoz-Ceballos iD, Á. García-Baños iD , C. Jaramillo-Molina
}

\begin{abstract}
These instructions give you guidelines for preparing papers for IEEE Transactions and Journals. Use this document as a template if you are using Microsoft Word 6.0 or later. Otherwise, use this document as an instruction set. Paper titles should be written in uppercase and lowercase letters, not all uppercase. Avoid writing long formulas with subscripts in the title; short formulas that identify the elements are fine (e.g., "Nd-Fe-B"). Do not write "(Invited)" in the title. Full names of authors are preferred in the author field, but are not required. Put a space between authors' initials. The abstract must be a concise yet comprehensive reflection of what is in your article. In particular, the abstract must be self-contained, without abbreviations, footnotes, or references. It should be a microcosm of the full article. The abstract must be between 150-250 words. Be sure that you adhere to these limits; otherwise, you will need to edit your abstract accordingly. The abstract must be written as one paragraph, and should not contain displayed mathematical equations or tabular material. The abstract should include three or four different keywords or phrases, as this will help readers to find it. It is important to avoid over-repetition of such phrases as this can result in a page being rejected by search engines. Ensure that your abstract reads well and is grammatically correct.
\end{abstract}

Index Terms - Cellular Automata, Crowd Simulation, Microscopic and Macroscopic Model, Pedestrian Movements.

Resumen - En este trabajo se presenta un modelo que usa un autómata celular para investigar la accesibilidad peatonal al interior del campus de la Universidad del Valle, que, actualmente, cuenta con $1.000 .000 \mathrm{~m} 2$, con un área construida de $164.469,35 \mathrm{~m} 2$ correspondiente a 56 edificios y con una movilidad de $\mathbf{1 5 . 5 0 0}$ peatones aproximadamente. El modelo es usado para simular el comportamiento del flujo peatonal sobre una red de corredores,

Este manuscrito fue enviado el 30 de noviembre de 2018 y aceptado el 28 de marzo, 2019.

Este proyecto fue patrocinado por la Universidad del Valle y Colciencias mediante la convocatoria 503-2010 del banco de anteproyectos de investigación científica o tecnológica con código de proyecto PRE00503029049 y titulado Modelamiento de la Accesibilidad Peatonal con Autómatas Celulares, este proyecto hace parte del programa nacional de electrónica telecomunicaciones e informática en las áreas de investigación de informática e ingeniería de transporte y fue desarrollado en cooperación entre los grupos de investigación GITTV (Grupo de Investigación en Transporte, Tránsito y Vías) y GEDI (Grupo de Investigación en Estudios Doctorales en Informática). que permite observar patrones emergentes de accesibilidad en las horas pico al interior del campus universitario, para así identificar, entender y encontrar las necesidades de movilidad de sus usuarios en los canales peatonales y facilitar la toma de decisiones en la mejora de la red peatonal para su ampliación o construcción. Para la construcción del modelo, se propuso una modificación de la vecindad de Moore y el desarrollo del prototipo se realizó mediante Desarrollo Dirigido por Comportamiento. El modelo representa el problema de movilidad peatonal en términos microscópicos y macroscópicos, y la implementación presenta dos entornos visuales: una interfaz gráfica en la que se ve la ejecución de la simulación y una interfaz modo carácter que solo muestra los resultados en un informe. Según los resultados de la implementación del modelo, los peatones en el autómata celular tienen el comportamiento esperado y resuelven los conflictos en la red de corredores de su origen a su destino, según las rutas definidas y en los tiempos esperados.

Palabras claves - Autómatas Celulares, Movimientos Peatonales, Modelo Microscópico y Macroscópico, Simulación de Multitudes.

\section{INTRODUCCIÓN}

A ctualmente, los campus universitarios son considerados como modelos de ciudades más pequeñas, que presentan en su interior los mismos comportamientos que las ciudades, tanto a nivel físico como social, político, económico y cultural, pero en una escala menor. La accesibilidad y la movilidad constituyen unos de los factores más representativos del funcionamiento del campus como una pequeña ciudad ya que estas inciden directamente en el comportamiento de las personas que conforman la comunidad universitaria.

Los problemas de la accesibilidad y la movilidad peatonal

V. M. Vargas-Forero es profesor tiempo completo de la Universidad del Valle (Cali, Colombia). (e-mail: victor.m.vargas@correounivalle.edu.co).

L. E. Muñoz-Ceballos es magister en ingeniería de la Universidad Valle (Cali, Colombia). (e-mail: luz.estela.munoz@correounivalle.edu.co).

Á. García-Baños es profesor tiempo completo de la EISC de la Universidad del Valle (Cali, Colombia). (e-mail: angel.garcia@ @ correounivalle.edu.co).

C. Jaramillo-Molina es profesor tiempo completo y director del Grupo de investigación en transporte, tránsito y vías - GITTV en la Universidad del Valle (Cali, Colombia). (e-mail: ciro.jaramillo@correounivalle.edu.co). 
son actualmente un campo muy interesante de investigación [1]-[3]. Las zonas peatonales en las que se realizan estos estudios son espacios públicos o privados cuyo propósito es permitir el tránsito de personas (peatones) a pie; estas zonas también son conocidas como pasillos, senderos, corredores, etc. Los lugares donde se han investigado y analizado los comportamientos de los peatones son terminales de transporte, aeropuertos, centros comerciales, parques, etc. y han sido objeto de estudio con el propósito de incorporar ingeniosas propuestas, conceptos y elementos urbanísticos que exigen las nuevas demandas de nuestra sociedad. Para realizar estos estudios, es necesario contar con herramientas automatizadas que provean información relevante para apoyar la toma de decisiones en la construcción de estos nuevos escenarios.

En la actualidad, existen diversos estudios que se han centrado en problemas de micro simulación [4], básicamente en problemas de evacuación [5] y [6]; algunos de estos involucran las características ambientales y los comportamientos de los vecinos [6]. En la literatura se encuentra mucha información de problemas de movilidad peatonal en términos microscópicos y muy poca en términos macroscópicos; la propuesta que se presenta en este artículo se encuentra enmarcada en ambos sentidos.

En los últimos años, se han mejorado los métodos y los algoritmos gracias a las posibilidades tecnológicas actuales. Las herramientas computacionales han posibilitado esta mejora, por lo tanto, en el contexto de la investigación, nos preguntamos si ¿Es posible simular el comportamiento de miles de peatones sobre una red de corredores, de manera que se puedan observar patrones emergentes de accesibilidad y así poder identificar sus características?

Esta investigación se centró en el campus universitario de Meléndez de la Universidad del Valle y se partió del estudio [7], en el que se definía que el campus está formado por 47 edificios construidos en un entorno paisajístico propuesto mediante espacios abiertos, patios, corredores, plazas, áreas deportivas y senderos; todo esto en función de la sociedad y sus demandas.

El campus universitario se ha convertido en un centro de desarrollo de la ciudad. Se ha ido urbanizando y complementando de servicios a través de todos estos años y ahora incluso es reconocido como jardín botánico y patrimonio arbóreo. Todas las vías de desplazamiento interno de la Universidad del Valle para los peatones están compuestas por senderos transitables ya determinados y otros senderos transitables y no transitables que no se encuentran determinados por la infraestructura de la administración de la Universidad. Estos senderos han emergido de las necesidades de los usuarios del campus universitario.

Para la administración de la Universidad del Valle, es importante contar con información que le ayude a entender y a encontrar las necesidades de movilidad de sus peatones (estudiantes, profesores, administrativos y particulares); entre estas se pueden identificar problemas de movilidad, densidad y flujo en los canales peatonales del campus. En este artículo se describe un modelo de autómata celular que permite investigar la accesibilidad peatonal al interior del campus universitario, y que contribuye a generar información necesaria para facilitar la toma de decisiones.

\section{METODOLOGÍA}

La construcción del modelo se inicia con el levantamiento y especificación de requerimientos mediante Desarrollo Dirigido por Comportamiento, de lo que se obtuvieron como resultado los test de comportamiento para cada una de las funcionalidades requeridas por el usuario final. Mediante estos test se realiza la especificación de requerimientos y se propone un primer modelo del autómata celular para modelar la accesibilidad peatonal al interior de la Universidad del Valle. Este modelo es analizado con el equipo de trabajo y, con base en este análisis, se procede a hacer correcciones de los problemas identificados. El paso anterior se repite hasta obtener un modelo adecuado a las necesidades, según la calibración del modelo.

\section{TRabajos Relacionados}

El estudio de la dinámica peatonal ha tenido gran desarrollo, principalmente, mediante modelos de autómatas celulares, debido a la eficiencia para modelar sistemas complejos. La rapidez de simulación y el número de personas con las que se pueden trabajar es mucho mayor que con otro tipo de modelos. A continuación, se mencionan algunos trabajos de investigación con este enfoque.

La simulación de autómatas celulares con diferentes velocidades, introducida por [8], presenta un modelo de autómata celular sin retroceso para la dinámica de peatones; en este se consideran las conductas humanas que pueden generar algunas situaciones complejas. El modelo puede simular el movimiento peatonal con velocidades diferentes, mediante la actualización de diferentes intervalos de tiempo. Ellos distinguen tres fases de los patrones de los peatones: la fase de movimiento libre, la fase de formación carril y la fase de no movimiento o detenido por algunos rangos de densidad. En la fase de formación de carril, se estudia el fenómeno de rozamiento, en el que los peatones caminan con velocidad inferior a través de un estrecho pasillo.

Hoy en día, hay una necesidad de crear diversos modelos que tienen en cuenta la escala microscópica de simulación. En [9] se presenta un modelo que describe la dinámica de peatones en una determinada área limitada. Los peatones están representados por elipses en una red cuadrada, lo que implica la necesidad de tener en cuenta algunas limitaciones geométricas para cada celda. En este modelo se introduce una idea innovadora sobre las distancias sociales.

El uso de multi-agentes en la simulación del flujo peatonal con autómatas celulares, descrito por [10], presenta un modelo básico multi-capa, en el que se procesa el movimiento humano. Ellos mencionan que usan un modelo de comportamiento para simular las acciones individuales de los peatones y usan un modelo de autómata celular para simular los movimientos de pequeña escala.

La teoría de los autómatas celulares como una aproximación 
para abordar la dinámica de peatones se presenta en [11], en el que se muestra un modelo de autómata celular bidimensional para la simulación de la dinámica de peatones. El modelo es extremadamente eficiente, permite simulaciones de multitudes e incluye interacciones sólo con el vecino más cercano; sin embargo, es capaz de reproducir efectos colectivos y la autoorganización que se encuentra en la dinámica peatonal. Esto se logra mediante la introducción de un campo de suelo, como unidad de interacción entre los peatones. En este artículo se centran en transiciones sólo entre células vecinas, tiene su propia dinámica (diffusion and decay) y puede ser cambiado por el movimiento de los peatones. Por lo tanto, el modelo usa una idea similar a la quimiotáxis, pero con los peatones siguiendo un rastro virtual en vez de un rastro químico. Su modelo utiliza simulación de Monte Carlo.

El modelo de klüpfeld [12], a diferencia de la mayor parte de trabajos, contempla una velocidad máxima mayor a uno, por lo que cada peatón puede avanzar más de una celda en cada paso de tiempo. Por su parte, en [13] se presenta una simulación de flujo peatonal bidireccional basado en autómatas celulares (CA). Este modelo tiene en cuenta dos aspectos: la dirección y el hábito al caminar de los peatones. La simulación utiliza parámetros dinámicos (parámetro de dirección, de vacío, de avance y de categoría) para simplificar tácticamente el proceso de toma de decisiones de los peatones en sus movimientos. Las relaciones de velocidad-densidad y flujo de densidad fueron estudiadas y analizadas.

Otro aspecto que se tiene en cuenta en las investigaciones es el de las colisiones [14]. El modelo dice que las reglas locales que rigen el movimiento de los peatones se determinan automáticamente sobre el proceso de simulación. En esta investigación, los autores introducen el concepto de "patrón de colisión" sobre un área de visión, y cada peatón se enseña a no chocar con otros. Este aspecto también es investigado por [15], en el que proponen la idea del "campo de piso". Las interacciones entre individuos para distancias cortas son de tipo repulsivas y en distancias grandes son de tipo atractivas. Así, los peatones mantienen su distancia y tienen la ventaja de seguir a otras personas con la misma dirección. El modelo maneja dos tipos de estados de ánimo: "feliz" o "infeliz". El estado feliz es para los peatones que tratan de moverse en su dirección deseada, mientras que los peatones con estado infeliz se mueven aleatoriamente. De esta manera, los peatones no bloquean el camino a otras personas, reduciendo así embotellamientos.

\section{RESULTADOS}

Nuestro modelo se basa en autómatas celulares y su propósito es simular el tráfico peatonal al interior del campus universitario ciudad Meléndez en la Universidad del Valle. A continuación, describimos el modelo de autómata celular:

El autómata celular se define formalmente como una tupla ( $\mathrm{G}, \mathrm{S}, \mathrm{N}, \mathrm{R})$, es decir un conjunto ordenado de objetos caracterizados por:
$(G):$ La rejilla

$(S)$ :Los estados que pueden tomar una celda

$(N):$ La vecindad

$(R)$ : Las reglas de transición

A continuación, se describe cada una de estas características para el proyecto de la accesibilidad peatonal con autómatas celulares.

- (G)rejilla o cuadrícula (grilla), cada cuadro es una celda que puede contener un peatón.

- Cada celda es cuadrada de aproximadamente 40 x 40 $\mathrm{cm} 2$.

- El número de peatones que puede estar en una celda es 0 o 1 .

- El autómata se define sobre una cuadrícula que representa el campus universitario y cuyas dimensiones son 2589 filas 1906 columnas.

- (S) conjunto finito de estados que puede tomar una celda.

A continuación, se presentan el conjunto de estados que puede tomar cada celda para el modelo propuesto. Para nuestro modelo, la definición de impedancia será entendida como la resistencia (disminución de velocidad) que tiene un peatón al desplazarse por una vía o camino, ocasionada por motivos de una rampa, escalera o dificultad de pasar por la vía.

“" Celda vacía, sendero no transitable por cualquier
concepto.
P Celda ocupada - peatón.
$0 \quad$ Celda vacía, sendero transitable sin impedancia.
1...n Celda vacía, sendero transitable con impedancia,
el valor de la impedancia depende del valor de n,
en el que 1 es la impedancia mínima que retrasa
al peatón un ciclo por cada movimiento. Para n
se retrasaría el peatón n ciclos por cada
movimiento.
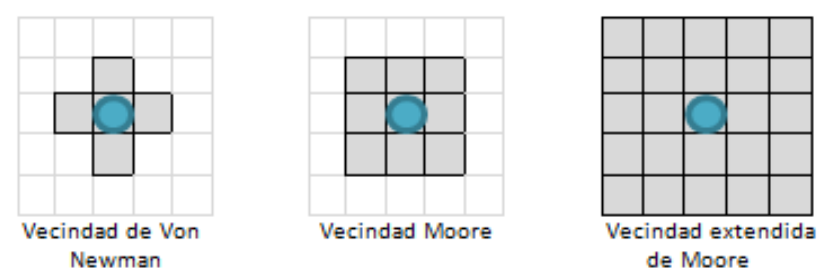

Fig. 1. Tipos de Vecindad.

- $\quad(\mathrm{N})$ :es la vecindad correspondiente a cada celda.

Para el proyecto, se toma como base la vecindad de Moore extendida, que es una extensión de la vecindad de Von Newman. Esta vecindad contiene celdas en la diagonal, como se puede ver en la Figura 1. En el literal A del numeral 4 se describen las modificaciones a la vecindad de Moore. 
- (R): reglas de transición que definen el modelo del autómata celular. En la sección C del numeral IV se describen las reglas de transición para el modelo propuesto.

\section{A. Modificaciones a la vecindad de Moore}

Las consideraciones para el modelo propuesto son: el alcance de la vecindad máxima es igual a 2 celdas y no se consideran todos los vecinos dentro de los movimientos posibles. Nuestro peatón no considera retroceder en su trayectoria de origen a destino, por lo que sólo realiza movimientos en un solo sentido.

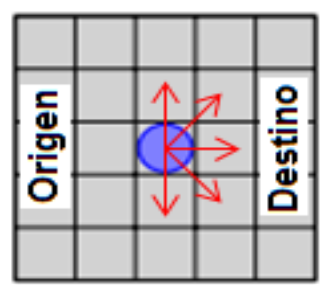

Fig. 2. Vecindad $\mathrm{V}_{1}$

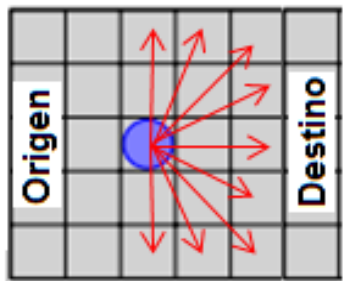

Fig. 3. Vecindad $\mathrm{V}_{2}$
En la Figura 2, se pueden ver las posibles opciones de vecindad para los peatones de vecindad de velocidad $1\left(\mathrm{~V}_{1}\right)$ y la Figura 3 , vecindad para peatones de velocidad $2\left(\mathrm{~V}_{2}\right)$.

\section{B. Movimientos de los peatones}


Fig. 4. Movimientos de los peatones.

Cada peatón tiene 8 movimientos posibles según su origen y destino; estos se definen en la Figura 4.

Estos movimientos los podemos clasificar en dos tipos: movimientos frontales (de arriba a abajo, abajo a arriba, izquierda a derecha y derecha a izquierda) y movimientos en diagonales (de inferior derecha a izquierda superior, izquierda

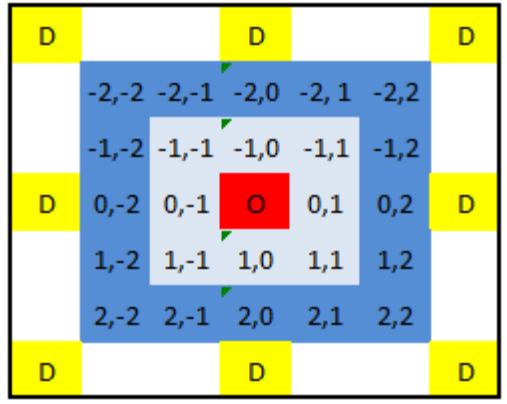

Fig. 5. Matriz de movimientos de los peatones

superior a derecha inferior, derecha superior a izquierda inferior e izquierda inferior a derecha superior).

En la Figura 5 se representan todos los movimientos posibles desde la posición origen $\mathrm{O}$ a cada destino $\mathrm{D}$ en la que cada movimiento está definido en azul claro para la velocidad $\mathrm{V}_{1} \mathrm{y}$ azul más oscuro para velocidad $\mathrm{V}_{2}$. Los valores para cada movimiento son valores de cambio en las coordenadas de los ejes de las abscisas y ordenadas del plano cartesiano. Usando estos valores, se construye la matriz de movimientos posibles para cualquier peatón MMp. Para la construcción de la matriz, se tomó como heurística que los movimientos se realizan o analizan primero de izquierda a derecha.

A continuación, se describe cada uno de los posibles movimientos para cada una de las velocidades.

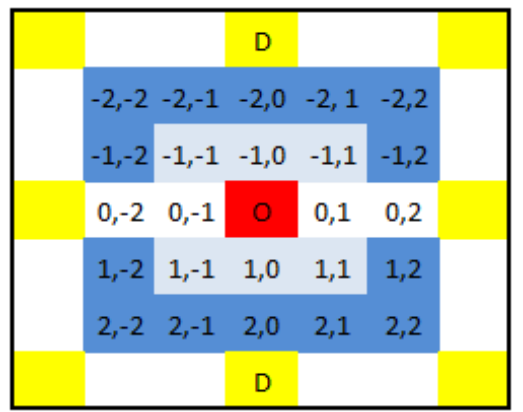

Fig. 6. Movimientos de un peatón de arriba/abajo, abajo/arriba

\section{1) Movimientos de un peatón en forma frontal}

La Fig. 6 ilustra los movimientos de forma frontal que puede realizar un peatón en dirección de arriba abajo y de abajo a arriba con velocidad $V_{1}$ y velocidad $V_{2}$, tal como se describe a continuación:

Los movimientos de arriba a abajo para el peatón con velocidad $\mathrm{V}_{1}$ están dados por:

$$
M M p_{1}=\left\{p_{1,0}, p_{1,1}, p_{1,-1}, p_{0,1}, p_{0,-1}\right\}
$$

De acuerdo a esto, un peatón $\mathrm{p}_{\mathrm{i}, \mathrm{j}}$ con velocidad $\mathrm{V}_{1}$ tiene movimiento de arriba abajo, Si $\ni x \mid x \in M M p_{1} \wedge x_{\text {estado }} \geq 0$

Los movimientos de abajo a arriba para el peatón con velocidad $\mathrm{V}_{1}$ están dados por:

$$
M M p_{2}=\left\{p_{-1,0}, p_{-1-, 1}, p_{-1,1}, p_{0,-1}, p_{0,1}\right\}
$$

De acuerdo a esto, un peatón $\mathrm{p}_{\mathrm{i}, \mathrm{j}}$ con velocidad $\mathrm{V}_{1}$ tiene movimiento de abajo a arriba, Si $\ni x \mid x \in M M p_{2} \wedge x_{\text {estado }} \geq 0$

Los movimientos de arriba a abajo para el peatón con velocidad $\mathrm{V}_{2}$ están dados por:

$$
M M p_{3}=\left\{p_{2,0}, p_{2,1}, p_{2,-1}, p_{2,2}, p_{2,-2}, p_{1,2}, p_{1,-2}, p_{0,2}, p_{0,-2}\right\}
$$

De acuerdo a esto, un peatón $\mathrm{p}_{\mathrm{i}, \mathrm{j}}$ con velocidad $\mathrm{V}_{2}$ tiene movimiento de arriba a abajo, Si $\ni x \mid x \in M M p_{3} \wedge x_{\text {estado }} \geq 0$

Los movimientos de abajo a arriba para el peatón con velocidad $\mathrm{V}_{2}$ están dados por:

$$
\begin{aligned}
& M M p_{4} \\
& =\left\{p_{-2,0}, p_{-2,-1}, p_{-2,1}, p_{-2,-2}, p_{-2,2}, p_{-1,-2}, p_{-1,2}, p_{0,-2}, p_{0,2}\right\}
\end{aligned}
$$




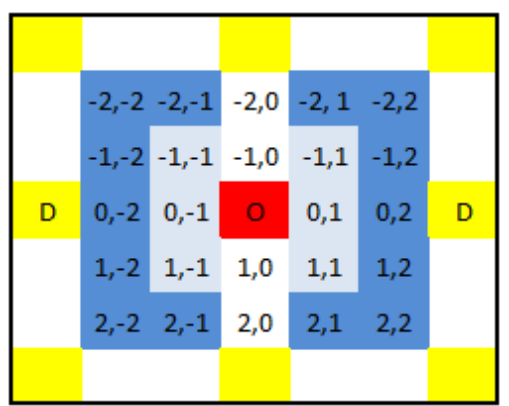

Fig. 7. Movimientos de un peatón de izquierda/derecha, derecha/izquierda

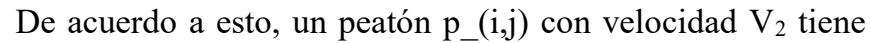
movimiento de abajo a arriba, Si $\ni x \mid x \in M M p_{4} \wedge x_{\text {estado }} \geq 0$

La Figura 7 ilustra los movimientos de forma frontal que puede realizar un peatón en dirección de izquierda a derecha y de derecha a izquierda con velocidad $V_{1}$ y velocidad $V_{2}$, tal como se describe a continuación:

Los movimientos de izquierda a derecha para el peatón con velocidad $\mathrm{V}_{1}$ están dados por:

$$
M M p_{5}=\left\{p_{0,1}, p_{-1,1}, p_{1,1}, p_{-1,0}, p_{1,0}\right\}
$$

De acuerdo a esto, un peatón $\mathrm{p}_{\mathrm{i}, \mathrm{j}}$ con velocidad $\mathrm{V}_{1}$ tiene movimiento de izquierda a derecha, Si $\ni x \mid x \in M M p_{5} \wedge x_{\text {estado }} \geq 0$

Los movimientos de derecha a izquierda para el peatón con velocidad $\mathrm{V}_{1}$ están dados por:

$$
M M p_{6}=\left\{p_{0,-1}, p_{1,-1}, p_{-1,-1}, p_{1,0}, p_{-1,0}\right\}
$$

De acuerdo a esto, un peatón $\mathrm{p}_{\mathrm{i}, \mathrm{j}}$ con velocidad $\mathrm{V}_{1}$ tiene movimiento de derecha a izquierda, Si $\ni x \mid x \in M M p_{6} \wedge x_{\text {estado }} \geq 0$

Los movimientos de izquierda a derecha para el peatón con velocidad $\mathrm{V}_{2}$ están dados por:

$$
M M p_{7}=\left\{p_{0,2}, p_{-1,2}, p_{1,2}, p_{-2,2}, p_{2,2}, p_{-2,1}, p_{2,1}, p_{-2,0}, p_{2,0}\right\}
$$

De acuerdo a esto, un peatón $\mathrm{p}_{\mathrm{i}, \mathrm{j}}$ con velocidad $\mathrm{V}_{2}$ tiene movimiento de izquierda a derecha, Si $\ni x \mid x \in M M p_{7} \wedge x_{\text {estado }} \geq 0$

Los movimientos de derecha a izquierda para el peatón con velocidad V2 están dados por:

$$
\begin{aligned}
& M M p_{8}= \\
& \left\{p_{0,-2}, p_{1,-2}, p_{-1,-2}, p_{2,-2}, p_{-2,-2}, p_{2,-1}, p_{-2,-1}, p_{2,0}, p_{-2,0}\right\}
\end{aligned}
$$

De acuerdo a esto, un peatón $\mathrm{p}_{\mathrm{i}, \mathrm{j}}$ con velocidad $\mathrm{V}_{2}$ tiene movimiento de derecha a izquierda, Si $\ni x \mid x \in M M p_{8} \wedge x_{\text {estado }} \geq 0$

\section{2) Movimientos de un peatón en forma diagonal}

La Figura 8 ilustra los movimientos de forma diagonal que puede realizar un peatón en dirección de derecha inferior a izquierda superior e izquierda superior a derecha inferior con

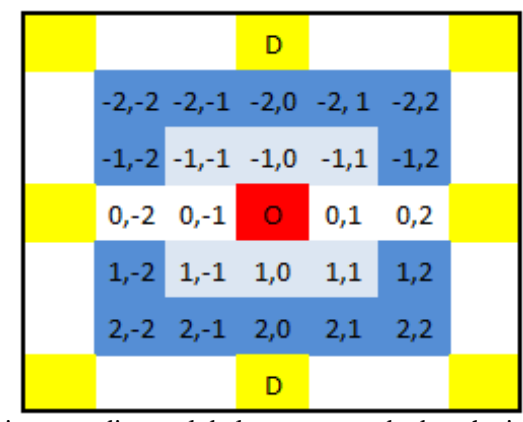

Fig. 8. Movimiento en diagonal de los peatones de derecha inferior/izquierda superior y de izquierda superior/derecha inferior

velocidad $V_{1}$ y velocidad $V_{2}$, tal como se describe a continuación:

Los movimientos de derecha inferior a izquierda superior para el peatón con velocidad $\mathrm{V}_{1}$ están dados por:

$$
M M p_{9}=\left\{p_{-1,-1}, p_{0,-1}, p_{-1,0}, p_{1,-1}, p_{-1,1}\right\}
$$

De acuerdo a esto, un peatón $\mathrm{p}_{\mathrm{i}, \mathrm{j}}$ con velocidad $\mathrm{V}_{1}$ tiene movimiento de derecha inferior a izquierda superior, Si $\ni x \mid x \in M M p_{9} \wedge x_{\text {estado }} \geq 0$

Los movimientos de izquierda superior a derecha inferior para el peatón con velocidad V1 están dados por:

$$
M M p_{10}=\left\{p_{1,1}, p_{0,1}, p_{1,0}, p_{-1,1}, p_{1,-1}\right\}
$$

De acuerdo a esto, un peatón $\mathrm{p}_{\mathrm{i}, \mathrm{j}}$ con velocidad $\mathrm{V}_{1}$ tiene movimiento de izquierda superior a derecha inferior, Si $\ni x \mid x \in M M p_{10} \wedge x_{\text {estado }} \geq 0$

Los movimientos de derecha inferior a izquierda superior para el peatón con velocidad $\mathrm{V}_{2}$ están dados por:

$$
\begin{aligned}
& M M p_{11} \\
& =\left\{p_{-2,-2}, p_{-1,-2}, p_{-2,-1}, p_{0,-2}, p_{-2,0}, p_{1,-2}, p_{-2,1}, p_{2,-2}, p_{-2,2}\right\}
\end{aligned}
$$

De acuerdo a esto, un peatón $\mathrm{p}_{\mathrm{i}, \mathrm{j}}$ con velocidad $\mathrm{V}_{2}$ tiene movimiento de derecha inferior a izquierda superior, Si $\ni x \mid x \in M M p_{11} \wedge x_{\text {estado }} \geq 0$

Los movimientos de izquierda superior a derecha inferior para el peatón con velocidad $\mathrm{V}_{2}$ están dados por:

$$
M M p_{12}=\left\{p_{2,2}, p_{1,2}, p_{2,1}, p_{0,2}, p_{2,0}, p_{-1,2}, p_{2,-1}, p_{-2,2}, p_{2,-2}\right\}
$$

De acuerdo a esto, un peatón $\mathrm{p}_{\mathrm{i}, \mathrm{j}}$ con velocidad $\mathrm{V}_{2}$ tiene movimiento de izquierda superior a derecha inferior, Si $\ni x \mid x \in M M p_{12} \wedge x_{\text {estado }} \geq 0$

La Figura 9 ilustra los movimientos de forma diagonal que puede realizar un peatón en dirección de derecha superior a izquierda inferior e izquierda inferior a derecha superior con velocidad $V_{1}$ y velocidad $V_{2}$, tal como se describe a continuación: 
Los movimientos de derecha superior a izquierda inferior para el peatón con velocidad $\mathrm{V}_{1}$ están dados por:

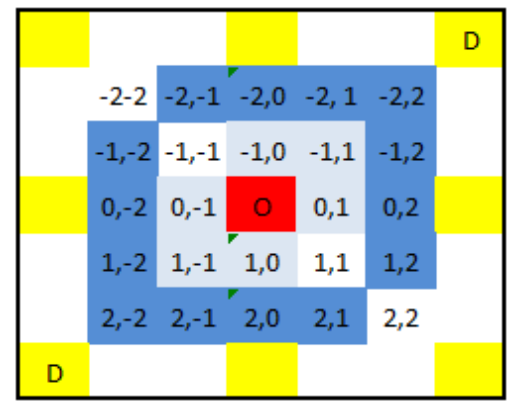

Fig. 9. Movimientos en diagonal de los peatones de derecha superior a izquierda inferior y de izquierda inferior a derecha superior

$$
M M p_{13}=\left\{p_{1,-1}, p_{1,0}, p_{0,-1}, p_{1,1}, p_{-1,-1}\right\}
$$

De acuerdo a esto, un peatón $\mathrm{p}_{\mathrm{i}, \mathrm{j}}$ con velocidad $\mathrm{V}_{1}$ tiene movimiento de derecha superior a izquierda inferior, Si $\ni x \mid x \in M M p_{13} \wedge x_{\text {estado }} \geq 0$

Los movimientos de izquierda inferior a derecha superior para el peatón con velocidad $\mathrm{V}_{1}$ están dados por:

$$
M M p_{14}=\left\{p_{-1,1}, p_{-1,0}, p_{0,1}, p_{-1,-1}, p_{1,1}\right\}
$$

De acuerdo a esto, un peatón $\mathrm{p}_{\mathrm{i}, \mathrm{j}}$ con velocidad $\mathrm{V}_{1}$ tiene movimiento de izquierda inferior a derecha superior, Si $\ni x \mid x \in M M p_{14} \wedge x_{\text {estado }} \geq 0$

Los movimientos de derecha superior a izquierda inferior para el peatón con velocidad $\mathrm{V}_{2}$ están dados por:

$$
\begin{aligned}
& M M p_{15} \\
& =\left\{p_{2,-2}, p_{2,-1}, p_{1,-2}, p_{2,0}, p_{0,2}, p_{2,1}, p_{-1,-2}, p_{2,2}, p_{-2,-2}\right\}
\end{aligned}
$$

De acuerdo a esto, un peatón $\mathrm{p}_{\mathrm{i}, \mathrm{j}}$ con velocidad $\mathrm{V}_{2}$ tiene movimiento de derecha superior a izquierda inferior, Si $\ni x \mid x \in M M p_{15} \wedge x_{\text {estado }} \geq 0$

Los movimientos de izquierda inferior a derecha superior para el peatón con velocidad $\mathrm{V}_{2}$ están dados por:

$$
\begin{aligned}
& M M p_{16} \\
& =\left\{p_{-2,2}, p_{-2,1}, p_{-1,2}, p_{-2,0}, p_{0,-2}, p_{-2,-1}, p_{1,2}, p_{-2,-2}, p_{2,2}\right\}
\end{aligned}
$$

De acuerdo a esto, un peatón $\mathrm{p}_{\mathrm{i}, \mathrm{j}}$ con velocidad $\mathrm{V}_{2}$ tiene movimiento de izquierda inferior a derecha superior, Si $\ni x \mid x \in M M p_{16} \wedge x_{\text {estado }} \geq 0$

\section{Las reglas de transición}

A continuación, se menciona el conjunto de reglas de transición para el modelo del autómata celular así:

- Todos los peatones se actualizan al tiempo en el mismo periodo de ciclo.

- Todos los movimientos de los peatones se realizan en el orden definido en la matriz de movimientos (ver Figura 5).

- $\quad$ Para que un peatón se mueva de posición, debe evaluar la matriz de movimiento y verificar el conjunto de todas las reglas.

- $\quad$ Si la celda a la que se dirige el peatón está ocupada, el peatón no se mueve.

- $\quad$ Si está libre y no hay otro peatón que se dirija a la misma celda, el movimiento se realiza.

- Si hay más de un peatón que se dirija a la misma celda, la toma cualquiera de los peatones; todos tienen la misma probabilidad.

- Los peatones tienen dos tipos de velocidad así:

○ $\quad \mathrm{V}_{1}=1$, el peatón se desplaza 1 celda por cada paso de tiempo.

- $\quad V_{2}=2$, el peatón se desplaza 2 celdas por cada paso de tiempo.

- $\quad$ Si un peatón no tiene celdas libres al usar velocidad 2, evalúa la posibilidad de movimiento en velocidad 1 , como se explica en el modelo.

- Las fronteras de nuestro modelo son fijas y están definidas por el área del campus universitario.

- El modelo contempla manejo de pendiente cuyo valor puede ir de 1 a $\mathrm{n}$. La pendiente representa el tipo de suelo (pavimento, tierra, escaleras, etc.).

- $\quad$ Las celdas con pendiente, según el tipo de pendiente (n), retrasan $n$ ciclos el movimiento del peatón.

Mediante estas reglas, el peatón sabe cómo moverse en un entorno local y dadas las posiciones de origen y destino de cada peatón, este se desplaza con un sentido determinado. El problema siguiente a resolver es cómo hacer para que el peatón no tome un camino incorrecto (sin salida) a lo largo de toda la ruta que debe transitar en el campus universitario. Para esto, se crea la definición de los caminos que debe tomar el peatón desde su origen hasta su destino. Dicha definición determina el camino que el peatón sigue, evitando así que tome un camino incorrecto. Esta definición se guarda en un archivo plano que contiene la posición o coordenada que debe seguir cada peatón a lo largo de la ruta, ejemplo:

Registro de una ruta determinada.

\section{(Ruta Origen Destino) \\ (1 1276,749,1276,760 864,746,864,763) \\ (2 1276,749,1276,760 864,746,864,763,864,763,1023,756)}

Ruta (color rojo) indica la identificación de una de las rutas del campus universitario; el origen (color azul) es un rectángulo definido por cuatro coordenadas mediante dos puntos, en esta área, el peatón puede ser generado, y, por último, el destino (color verde) tiene mínimo cuatro coordenadas para generar el área de llegar a su destino, pero puede tener más puntos (parejas de coordenadas) que determinan destinos parciales para cada peatón para esta ruta determinada. Como podemos observar, en la ruta 1 no hay destinos parciales, mientras que en la ruta 2 , 
existen dos destinos parciales $(864,763,1023,756)$ que el peatón debe transitar antes de llegar a su destino final.

\section{CONCLUSIONES}

Uno de los principales resultados a resaltar en el presente trabajo es que se abordaron simultáneamente los problemas de simulación micro y macroscópicos usando autómatas celulares; esta propuesta es un primer intento de abordar este problema desde esta perspectiva. El proyecto desarrolló su solución utilizando como proceso de desarrollo de software BDD (por sus siglas en inglés: Behavior-Driven Development), obteniendo como resultado los tests de comportamiento para cada una de las funcionalidades requeridas por el usuario final.

Durante la calibración del modelo, se realizaron varias pruebas -que serán objeto de otro artículo- y el propósito era lograr que el modelo implementado se adaptara a información específica del mundo real. Esta información provino de las caracterizaciones de la oferta y la demanda de transporte peatonal, que fue fruto de un trabajo realizado en el laboratorio de tránsito y transporte GITTV en la tesis de pregrado de la estudiante Liliana Rodríguez Saac [16].

La simulación se concentró en la franja horaria del mediodía, entre las 12:00 horas y las 14:30 horas, ya que esta es la que más peatones moviliza. De esta franja, las zonas analizadas de su respectivo origen a su destino con más peatones fueron: cafetería, ciencias, ingenierías y portería peatonal, ya que fueron las zonas con mayor atracción y producción de peatones en la tesis propuesta por Liliana Rodríguez Saac [16]. Como podemos ver a continuación, los peatones que ingresan al sistema en un número de ciclos determinado coinciden con los aforos realizados por el grupo GITTV, al igual que los peatones que salen del sistema.

\begin{tabular}{cccc}
\hline \hline & \multicolumn{3}{c}{ Peatones que ingresan al sistema } \\
& 1600 ciclos & 6000 ciclos & GITTV \\
Ciencias & 711 & 934 & 1360 \\
Ingenierías & 561 & 910 & 929 \\
\hline \hline
\end{tabular}

Hay que recordar que los ciclos son la unidad de tiempo de los movimientos de cada peatón y son equivalentes a un paso de un peatón en el sistema. En la columna GITTV están todos los peatones que salieron e ingresaron en el intervalo del mediodía según los resultados presentados en [16] y 6000 ciclos serían un equivalente a 100 minutos aproximadamente.

\begin{tabular}{cccc}
\hline \hline & \multicolumn{3}{c}{ Peatones que salan del sistema } \\
& 1600 ciclos & 6000 ciclos & GITTV \\
Ciencias & 31 & 843 & 1145 \\
Ingenierías & 87 & 784 & 425 \\
\hline \hline
\end{tabular}

El simulador provee 2 entornos visuales, un entorno gráfico en el que se puede ver la cuadrícula y la red peatonal del campus, en la que se puede apreciar dinámicamente cómo se van generando los peatones y cómo se desplazan durante la ejecución de la simulación. Esta interfaz requiere de un equipo con muy buenas características para su funcionamiento (ver
Figura 10). La segunda opción es un entorno en el que los resultados son presentados en la pantalla y se genera un archivo plano con el seguimiento de toda la ejecución (ver Figura 11); sus ventajas, con respecto a la interfaz gráfica, es que se ejecuta mucho más rápido y no requiere de un equipo con tan altas

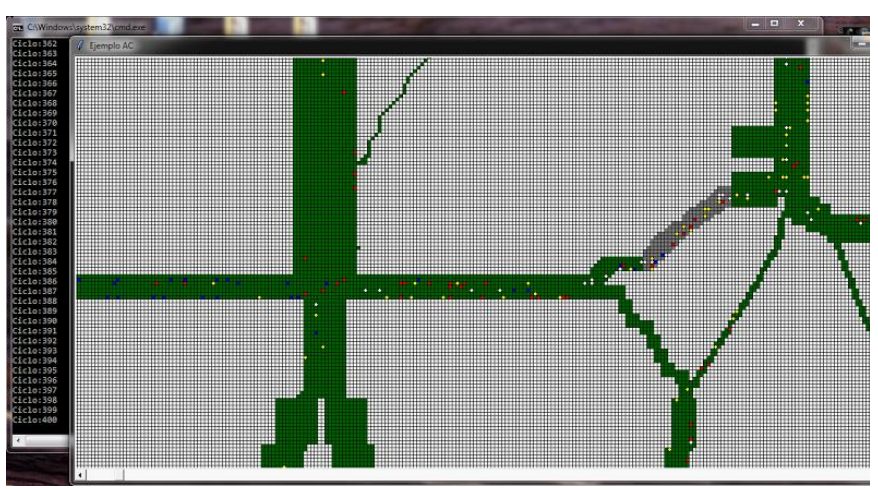

Fig. 10. Entorno gráfico

características.

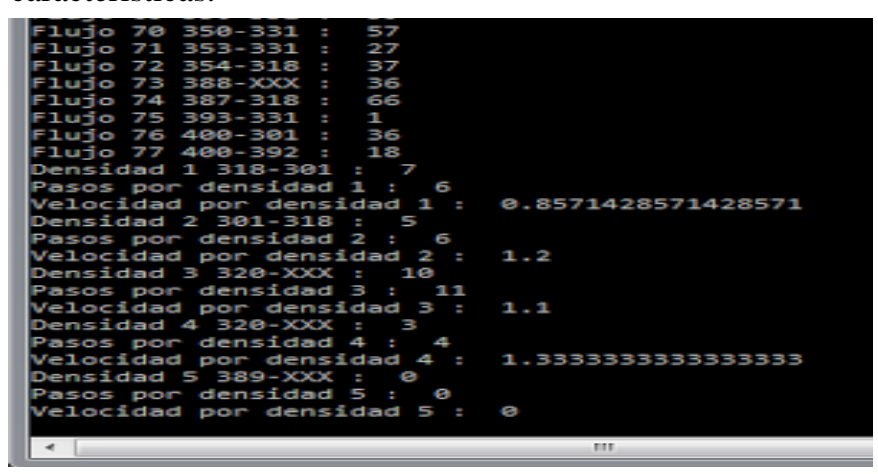

Fig. 11. Entorno no gráfico

\section{REFERENCES}

[1] N. M. Gonçalves and A. N. Basantes, "Assessment the Pedestrian Accessibility in the BRT Stations in Two Cities of Latin America (breakout presentation)," J. Transp. Heal., vol. 7, pp. S76-S77, 2017. DOI: $10.1016 /$ j.jth.2017.11.124

[2] B. Y. Chen, Y. Wang, D. Wang, Q. Li, W. H. K. Lam, and S. L. Shaw, "Understanding the impacts ofhuman mobility on accessibility usingmassivemobile phone tracking data," Ann. Am. Assoc. Geogr., vol. 108, no. 4, pp. 1115-1133, 2018. DOI: $10.1080 / 24694452.2017 .1411244$

[3] H. Qin, K. M. Curtin, and M. T. Rice, "Pedestrian network repair with spatial optimization models and geocrowdsourced data," GeoJournal, vol. 83, no. 2, pp. 347-364, 2018. DOI: 10.1007/s10708-017-9775-x

[4] P. G. Gipps and B. Marksjö, "A micro-simulation model for pedestrian flows," Math. Comput. Simul., vol. 27, no. 2-3, pp. 95105, Apr. 1985. DOI: 10.1016/0378-4754(85)90027-8

[5] B. Gudowski and J. Wạs, "Some criteria of making decisions in pedestrian evacuation algorithms," in Proceedings - 6th International Conference on Computer Information Systems and Industrial Management Applications, CISIM 2007, 2007, pp. 9396. DOI: 10.1109/CISIM.2007.61 
[6] J. Wąs, "Cellular automata model of pedestrian dynamics for normal and evacuation conditions," in Proceedings - 5th International Conference on Intelligent Systems Design and Applications 2005, ISDA '05, 2005, vol. 2005, pp. 154-159. DOI: 10.1109/ISDA.2005.31

[7] P. Buitrago and J. Kattán, Universidad del Valle arquitectura para la educación, Primera. Santiago de Cali: Universidad del Valle, 2011.

[8] W. G. Weng, T. Chen, H. Y. Yuan, and W. C. Fan, "Cellular automaton simulation of pedestrian counter flow with different walk velocities," Phys. Rev. E - Stat. Nonlinear, Soft Matter Phys., vol. 74, no. 3, p. 36102, Sep. 2006. DOI: 10.1103/PhysRevE.74.036102

[9] J. Wąs, B. Gudowski, and P. J. Matuszyk, "Social distances model of pedestrian dynamics," in Cellular Automata, 2006, pp. 492-501. DOI: $10.1007 / 11861201 \_57$

[10] S. Sarmady, F. Haron, and A. Z. H. Talib, "Multi-agent simulation of circular pedestrian movements using cellular automata," in Proceedings - 2nd Asia International Conference on Modelling and Simulation, AMS 2008, 2008, pp. 654-659. DOI: 10.1109/AMS.2008.104

[11] C. Burstedde, A. Kirchner, K. Klauck, A. Schadschneider, and J. Zittartz, "Cellular Automaton Approach to Pedestrian Dynamics Applications,” Pedestr. Evacuation Dyn., pp. 75-86, 2001. DOI: citeulike-article-id:7115800

[12] H. Klupfel, A Cellular Automaton Model for Crowd Movement. GRIN Verlag, 2003.

[13] H. Yue, H. Guan, J. Zhang, and C. Shao, "Study on bi-direction pedestrian flow using cellular automata simulation," Phys. A Stat. Mech. its Appl., vol. 389, no. 3, pp. 527-539, Feb. 2010. DOI: 10.1016/j.physa.2009.09.035

[14] K. Narimatsu, T. Shiraishi, and S. Morishita, "Acquisition of Local Neighbor Rules in the Simulation of Pedestrian Flow by Cellular Automata," in Cellular Automata, 2004, pp. 211-219. DOI: 10.1007/978-3-540-30479-1_22

[15] C. Burstedde, K. Klauck, A. Schadschneider, and J. Zittartz, "Simulation of pedestrian dynamics using a two-dimensional cellular automaton," Phys. A Stat. Mech. its Appl., vol. 295, no. 34, pp. 507-525, Jun. 2001. DOI: 10.1016/S0378-4371(01)00141-8

[16] L. Rodríguez Saac, "Modelo de seguimiento de la movilidad peatonal en la universidad del valle sede Meléndez," Tesis de grado, 2012. [Online].

Available: http://bibliotecadigital.univalle.edu.co/bitstream/10893/9010/1/CB -0470310.pdf. [Accessed: 01-Jul-2018].

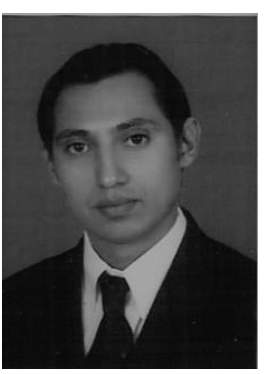

Víctor Manuel Vargas-Forero nació en Tuluá, Valle del Cauca, Colombia. Recibió su título de Tecnología en Sistemas de Información de la Universidad del Valle en 1997, su grado de Ingeniero en Sistemas en el 2001 de la Universidad Antonio Nariño, realizó y terminó especializaciones en Auditoría de Sistemas y en Procesos para el Desarrollo de Software en el 2002 y 2003, respectivamente, y en el 2011 obtuvo título de Maestría en Ingenierías con Énfasis en Ciencias de la Computación en la Universidad del Valle. Actualmente se desempeña como profesor tiempo completo en la Universidad del Valle, Colombia. Sus áreas de interés son los lenguajes de programación, la ingeniería de software y la inteligencia artificial.

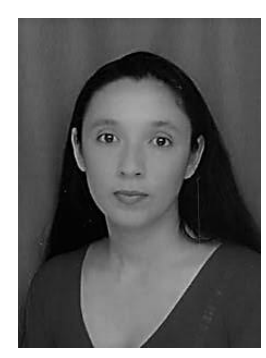

Luz Estela Muñoz-Ceballos nació en San Martín, Meta, Colombia. Recibió su título de Ingeniera en Sistemas en el 2007 de la Universidad del Valle y en el 2017 obtuvo título de Maestría con énfasis en Ingeniería de Sistemas también de la Universidad del Valle. Ha sido profesora de la Universidad del Valle y Universidad ICESI de Cali, Colombia y actualmente se encuentra vinculada como profesora tiempo completo de la Universidad Autónoma de Occidente de Cali, Colombia. Sus áreas de interés es el diseño de software multimedial y la inteligencia artificial.

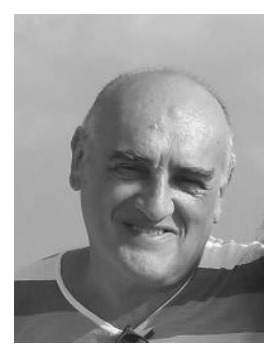

Ángel E. García Baños nació en Madrid, España y es profesor de la Universidad del Valle desde 1993 en las áreas de ingeniería electrónica y actualmente en computación. Obtuvo su diploma de Ingeniero de Telecomunicación en la ETSIT de la UPM (Madrid, España) en 1985 y el Doctorado en la UPV (Valencia, España). Pertenece al grupo GUIA y al laboratorio EVALAB. Como profesor titular de la Escuela de Ingeniería de Sistemas y Computación trabaja en sistemas complejos, vida artificial, computación evolutiva, inteligencia artificial y consciencia artificial.



Ciro Jaramillo Molina nació en Cali, Valle del Cauca, Colombia. Es Ingeniero Civil por la Universidad del Valle en 1989. Obtuvo su Diploma de estudios avanzados DEA y de Doctorado ambos en Ingeniería de Transporte por la Universidad Politécnica de Valencia, España en 2004 y 2009 respectivamente. Realizo estudios de 
Posdoctorado en Urbanismo y Ordenación del Territorio de la Universidad de Granada, España entre 2010 y 2011. Es Profesor Asociado de tiempo completo y Director del Grupo de investigación en transporte, tránsito y vías - GITTV en la Universidad del Valle en Cali, Colombia. Sus áreas de investigación son la accesibilidad y la equidad territorial y la siniestralidad vial y el análisis espacial. 